\title{
Simulation numérique de la propagation de fissure dans les superalliages monocristallins
}

\author{
Simulation numérique de la propagation de fissure \\ dans les monocristaux
}

\author{
Jean-Luc Bouvard — Jean-Louis Chaboche — Frédéric Feyel \\ Franck Gallerneau
}

DMSE/LCME ONERA

29 av. de la div. Leclerc F-92322 Châtillon cedex

jean-luc.bouvard@onera.fr

RÉSUMÉ. L'objet de cette étude est de développer une méthodologie de simulation numérique de propagation de fissure par fatigue, en appliquant une approche couplée. On s'intéresse plus particulièrement à l'utilisation d'éléments d'interface cohésifs, avec une loi de comportement spécifique, afin de simuler, sans discontinuité, et avec un caractère prédictif l'avancée d'une fissure. Nous présentons, tout d'abord, le modèle d'endommagement utilisé que nous validons par la suite grâce à une étude de convergence de la solution avec la taille de maille. Nous appliquons le modèle développé au cas d'une éprouvette préfissurée en superalliages monocristallin. Enfin, nous définissons la méthode d'identification des paramètres du modèle de zone cohésive sur la base des essais de propagation de fissure conduits en régime de fatigue pure.

ABSTRACT. In this study, a numerical method is developped to simulate fatigue crack growth. This method is based on a numerical coupled analysis using a cohesive zone modelling under cyclic loading in order to develop a coupled predictive approach of the crack growth. First, we present this damage law, then its validation by a convergence study of the solution with mesh size. We apply this model to the crack growth calculations in the case of a smooth specimen with a precracking made with a single crystal superalloy. Finally, we define the method to calibrate the cohesive zone model parameters on the base of experimental tests performed in pur fatigue regime.

MOTS-CLÉS : méthode éléments finis, éléments cohésifs, propagation de fissure, fatigue.

KEYWORDS: finite element method, cohesive zone elements, crack growth, fatigue.

DOI:10.3166/REMN.16.845-863 @ 2007 Lavoisier, Paris

REMN - 16/2007. Modélisations numériques en mécanique, pages 845 à 863 


\section{Introduction}

Les besoins en dimensionnement de composants critiques de turbomachines aéronautiques ont conduit au développement de différents modèles de prévision de durée de vie, soit en termes d'amorçage (fissure macroscopique typiquement de 300 microns de long), soit en termes de propagation de fissure longue. Les enjeux sont multiples : conception optimisée, augmentation du potentiel et de la fiabilité des matériels en service. L'objectif consiste à mieux maîtriser les outils prévisionnels pour la propagation des fissures dans le régime dit des « fissures courtes » (de 300 microns à $1 \mathrm{~mm}$ ), applicables après avoir estimé la durée de vie à amorçage de la fissure macroscopique par des modèles d'endommagement (Chaboche et al., 2001). Des modèles phénoménologiques, basés sur les travaux pionniers de Paris et al. (1961), reliant la vitesse de fissuration, da/dN à l'amplitude du facteur d'intensité de contraintes $\Delta K_{\text {eff }}=K_{\text {max }}-K_{\text {ouv }}$ (où $K_{\text {ouv }}$ est le facteur d'intensité de contraintes à ouverture de fissure), ont fourni des outils d'analyse très performants. La loi de Paris permet de décrire de manière fiable des données expérimentales de fissuration mais cela avec les hypothèses suivantes : plasticité confinée en pointe de fissure, amplitude de chargement constant et régime de fissures longues. Des extensions de la loi de Paris ont été proposées afin de prendre en compte les effets de rapport de charge (R), les seuils limites de propagation de fissure... Mais, dans le régime dit de fissures courtes, pour lequel l'hypothèse de plasticité confinée n'est plus respectée (vis-à-vis de la taille de la fissure), les vitesses de fissuration sont encore sous-estimées et difficilement prédites.

Dans cette étude, nous adoptons une approche de type zone cohésive afin de simuler de manière prédictive la propagation de fissure, notamment en régime de fissures courtes. Les modèles de zone cohésive sont caractérisés par un processus d'endommagement continu jusqu'à rupture finale de l'élément. De nombreux modèles de zone cohésive ont été proposés et appliqués avec succès pour décrire la fissuration sous traction monotone (Dugdale, 1960; Barenblatt, 1962; Needleman, 1987). Mais l'utilisation de tels modèles dans le cas de la fatigue aboutit à des arrêts de la fissure, consécutifs à la redistribution des contraintes en pointe de fissure. Des modèles spécifiques de zone cohésive doivent donc être développés pour simuler la propagation de fissures sous chargement cyclique. Cet article fait grandement référence aux articles suivants, (De-Andres et al., 1999; Nguyen et al., 2001; Yang et al., 2001; Tate, 2002) en particulier celui de Roe et al. (2003). Nous présentons, dans un premier temps, le modèle d'endommagement développé sous chargement cyclique. Puis, une étude de convergence de la solution en fonction de la taille de maille est présentée afin de s'assurer de la non-dépendance au maillage des éléments d'interface. Enfin, nous nous intéressons au cas plus complexe de l'avancée de fissure dans une éprouvette préfissurée en superalliage monocristallin, à haute température, sous chargement de fatigue cyclique ainsi qu'à la calibration du modèle de zone cohésive sur la base de résultats d'essais disponibles en régime de fatigue pure. 


\section{Présentation du modèle d'endommagement}

\subsection{L’approche zone cohésive}

L'approche zone cohésive permet d'envisager des situations mécaniques très complexes, notamment dans le domaine de l'analyse mécanique de l'amorçage et de la propagation de fissures, tout en permettant une description fine des mécanismes physiques à l'échelle locale. L'évolution de la fissuration est décrite grâce à des critères locaux, souvent énergétiques, et en termes de relation entre la contrainte (appliquée sur les lèvres de la fissure) $\mathrm{T}$ et la discontinuité de déplacement $[u]=u^{+}-u^{-}$, où $u^{+}$et $u^{-}$sont les déplacements respectifs de chaque lèvre de la fissure (figure 1).

Le modèle, présenté dans cet article, décrit l'évolution du comportement d'un élément d'interface dans un matériau déformable initialement vierge, qui, soumis à des chargements complexes, va s'endommager et se fissurer.

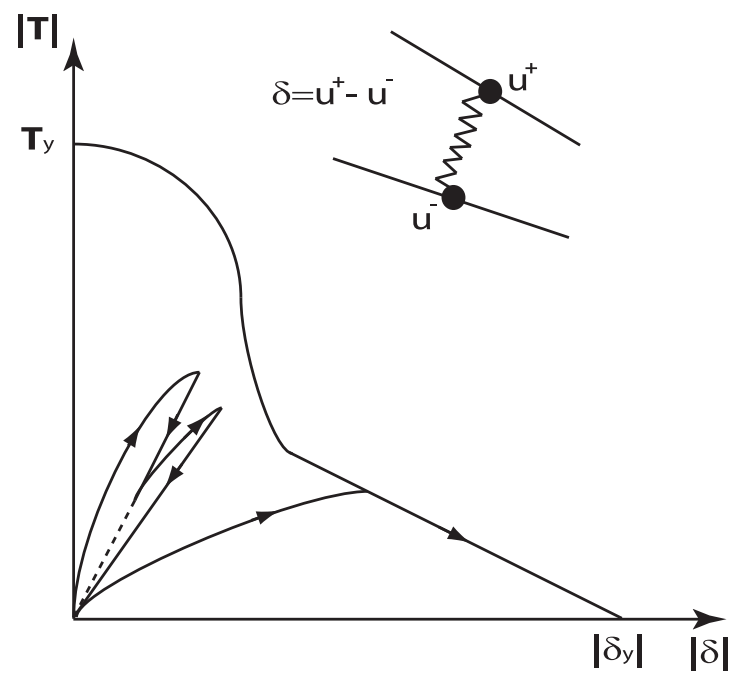

Figure 1. Modèle de zone cohésive

Dans cette section, nous explicitons une notion importante inhérente à l'utilisation des éléments de zone cohésive : la notion de «process zone ». Nous nous référons pour cela à un article de Chandra (Chandra et al., 2002) qui développe les notions de «Wake Zone » et de « Forward Zone» (figure 2). La process zone est la région dans laquelle les éléments de zone cohésive sont actifs, i.e., du point $\mathrm{A}$ où $\delta=u_{y}=0+\mathrm{au}$ point $\mathrm{E}$ de complète séparation de l'élément pour $\delta=\delta_{\text {sep }}$. Chandra propose de définir le point $\mathrm{C}\left(T=T_{\max }\right)$ comme étant relatif à la pointe de la fissure. En utilisant cette définition, la forward zone et la wake zone peuvent être aisément caractérisées comme cela est illustré sur la même figure. Cette notion de process zone est très importante 
car le nombre d'éléments de zone cohésive actifs dans cette région est un facteur prédominant pour une description correcte des champs de contraintes en pointe de fissure.

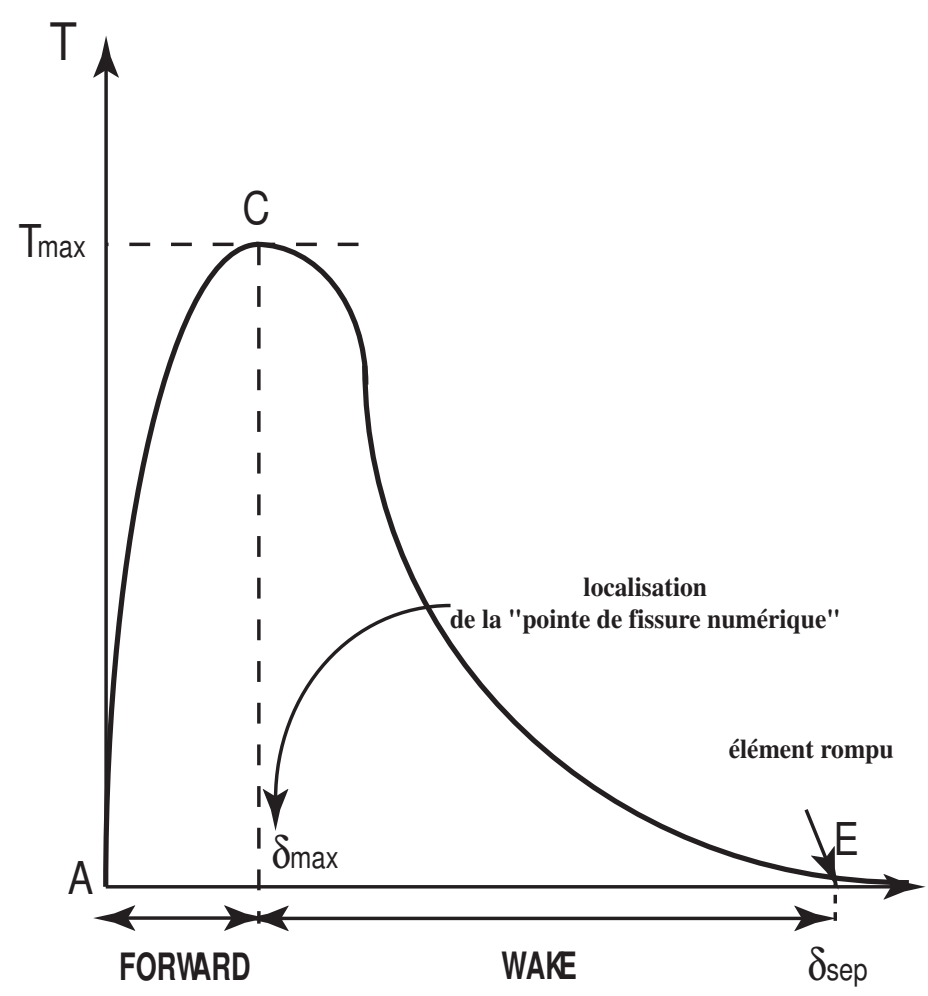

Figure 2. Comportement d'un modèle de zone cohésive sous chargement monotone

\subsection{Description thermodynamique du modèle de zone cohésive en fatigue pure}

La loi de comportement des modèles de zone cohésive est définie par une relation entre la force de traction, $T$, et le déplacement à ouverture de l'élément, $U$. Le modèle de zone cohésive, développé pour décrire la fissuration du matériau en régime de fatigue, est établi sur la base d'une fonction potentielle, $\phi$. Le potentiel d'état thermodynamique peut être postulé comme étant une fonction de différentes variables d'état, l'endommagement $\mathrm{D}$, le déplacement tangentiel $U_{T}$ et le déplacement à ouverture $U_{N}$ :

$$
\Phi=\frac{1}{2}(1-D) k_{N}\left[\frac{\left\langle U_{N}\right\rangle^{2}}{\delta_{0}}+\alpha \frac{U_{T}^{2}}{\delta_{0}}\right]
$$




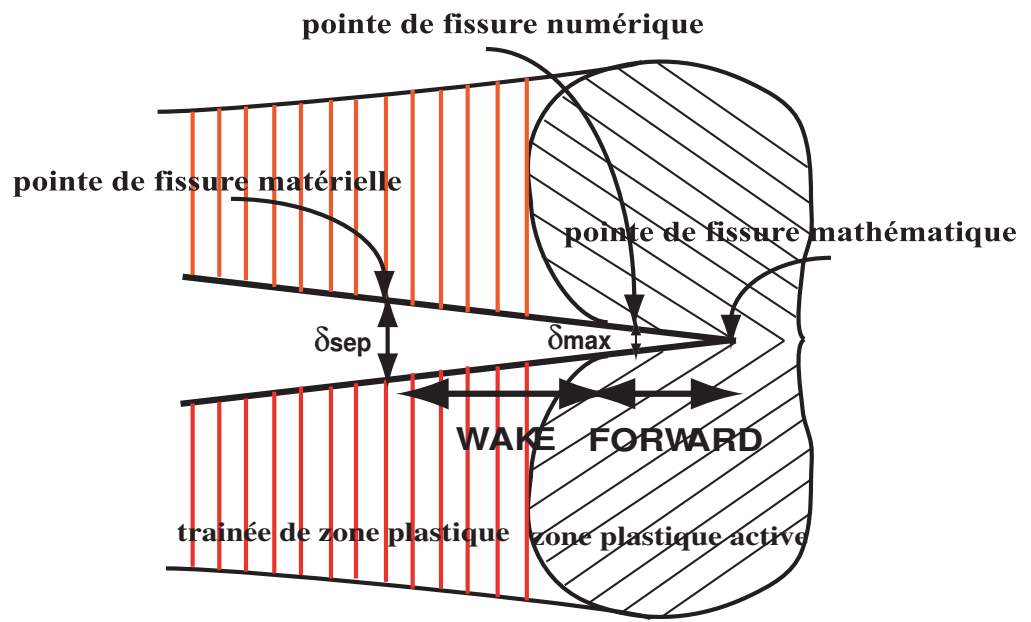

Dissipation intrinsèque

Dissipation extrinsèque

Figure 3. Description de la process zone

L'équation [1] est normée par l'introduction d'un déplacement constant $\delta_{0}$. Les paramètres matériaux sont la rigidité initiale de l'élément de zone cohésive donnée par le rapport $\frac{k_{N}}{\delta_{0}}$, la constante $\alpha$ et $\mathrm{D}$, la variable d'endommagement, égale à 0 pour un élément de zone cohésive non endommagé et atteignant une valeur de 1 pour un élément rompu.

Nous en déduisons alors les forces de traction normales et tangentielles, $T_{N}$ et $T_{T}$, qui dérivent du potentiel d'état. Nous avons choisi de caractériser le modèle de zone cohésive par une loi de comportement dans laquelle la force de traction est une fonction linéaire du déplacement à ouverture de l'élément, tandis que l'énergie de dissipation est prise en compte par l'introduction d'une variable d'endommagement non linéaire $\mathrm{D}$.

$$
\left\{\begin{array}{l}
T_{T}=\frac{\partial \Phi}{\partial U_{T}}=\alpha k_{N}(1-D) \frac{U_{T}}{\delta_{0}} \\
T_{N}=\frac{\partial \Phi}{\partial U_{N}}=k_{N}(1-D) \frac{\left\langle U_{N}\right\rangle}{\delta_{0}}
\end{array}\right.
$$

La force thermodynamique associée à l'endommagement cumulé durant les cycles de fatigue est définie par : 


$$
Y=-\frac{\partial \Phi}{\partial D}=\frac{1}{2} k_{N}\left[\frac{\left\langle U_{N}\right\rangle^{2}}{\delta_{0}}+\alpha \frac{U_{T}^{2}}{\delta_{0}}\right]
$$

Motivés par la formulation de Roe et Siegmund (Roe et al., 2003), nous avons choisi une cinétique d'endommagement caractérisée par une fonction $\dot{D}=$ $\dot{D}(D, Y, \dot{U})$. La variable d'endommagement est définie par une loi d'évolution qui prend en compte l'accumulation d'endommagement durant les phases de chargement et un endommagement constant durant les phases de décharge. La loi d'endommagement suivante est donc adoptée :

$$
\left\{\begin{array}{l}
\dot{D}=A \delta_{0}(1-D)^{m} H\left(\sqrt{Y}-\sqrt{Y_{0}}\right)^{n} \frac{\|\dot{U}\|}{\delta_{0}} \quad \text { si } \dot{Y} \geq 0 \\
\dot{D}=0 \quad \text { sinon }
\end{array}\right.
$$

où $\mathrm{A}$ est une constante contrôlant l'accumulation d'endommagement. $\|U\|$ est la norme du déplacement égale à $\sqrt{\frac{\left\langle U_{N}\right\rangle^{2}}{\delta_{0}}+\alpha \frac{U_{T}^{2}}{\delta_{0}}}, \dot{U}$ la vitesse du déplacement à ouverture de l'élément cohésif. $m$ et $n$ sont des constantes contrôlant la vitesse des mécanismes d'endommagement. $Y_{0}$ est le seuil de non-endommagement de l'élément et $\mathrm{H}$ désigne la fonction d'Heaviside.

La figure 4 illustre la réponse du modèle d'endommagement soumis à un chargement cyclique. Durant le premier chargement, caractérisé par un fort niveau, le damage locus est atteint (décrivant la réponse du modèle de zone cohésive pour un chargement monotone). Puis, pour un chargement cyclique à rapport de charge non nul, le modèle décrit des boucles d'hystérésis caractérisant les charges/décharges entre deux niveaux minimal et maximal de contrainte. Soumis à des chargements cycliques, l'élément d'interface s'endommage pendant la charge, mais pas en décharge. La rigidité de l'élément diminue avec l'accumulation de l'endommagement.

\section{Validation de l'approche adoptée et simulation de la propagation de fissure dans le cas d'une éprouvette préfissurée monocristalline}

\subsection{Géométrie du problème traité}

Dans le cadre de cette étude, nous considérons une éprouvette préfissurée (SEN), illustrée sur la figure 5, comportant une fissure initiale de longueur $a_{0}$. Cette éprouvette est orientée suivant $<001>$ et la direction du front de propagation de la fissure étudiée est $<100>$. La discrétisation par éléments finis prend en compte les symétries de chargement et de géométrie. Les détails du maillage en pointe de fissure sont donnés sur la figure 6. 


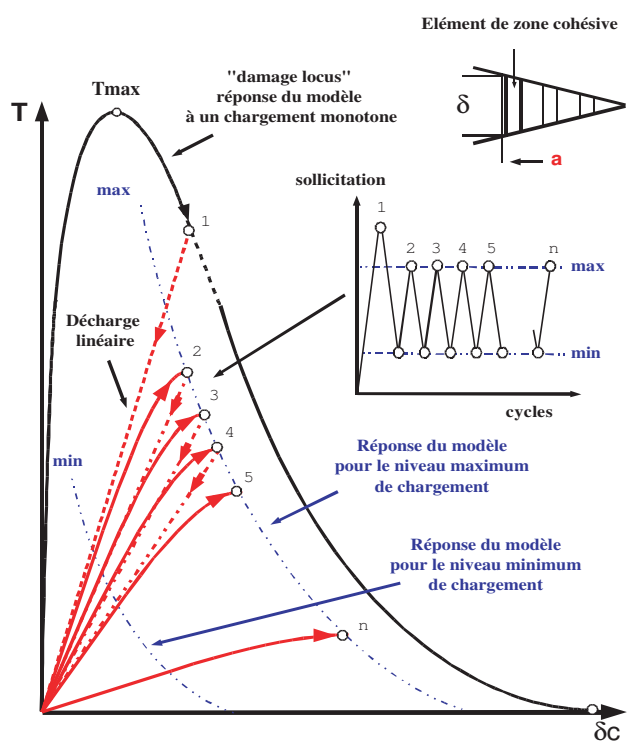

Figure 4. Réponse du modèle de zone cohésive avec accumulation d'endommagement durant la charge

Les éléments volumiques choisis sont des éléments triangulaires linéaires et les analyses éléments finis sont réalisées sous les hypothèses des déformations planes. Les éléments de zone cohésive sont des éléments quadrangles linéaires possédant deux points de Gauss. Un zoom de la zone de maillage raffinée montre une boîte régulièrement maillée où sont situés les éléments de zone cohésive. Le chemin de fissuration est supposé prédéfini le long de la ligne de symétrie. Le trajet de fissuration a été défini conformément aux observations de propagation de fissures expérimentales. Ce chemin de fissuration est modélisé par des éléments de zone cohésive. Différentes tailles d'éléments sont considérées dans cette étude.

Concernant les premiers calculs, la prise en compte de la préfissure a été réalisée en considérant une préfissure « élastique ». Cependant, on peut remarquer sur la figure 7 la présence d'une zone de très forte localisation des déformations plastiques au tout début de la simulation, caractéristique de l'amorçage de la fissure. Afin de diminuer cette localisation et d'avoir une bonne estimation de la trainée de zone plastique consécutive à la préfissuration, la simulation de celle-ci est réalisée en utilisant une méthode de relachement de nœuds (Prigent, 1993; Pommier, 1995), jusqu'à une longueur de préfissure donnée $a_{0}$. 


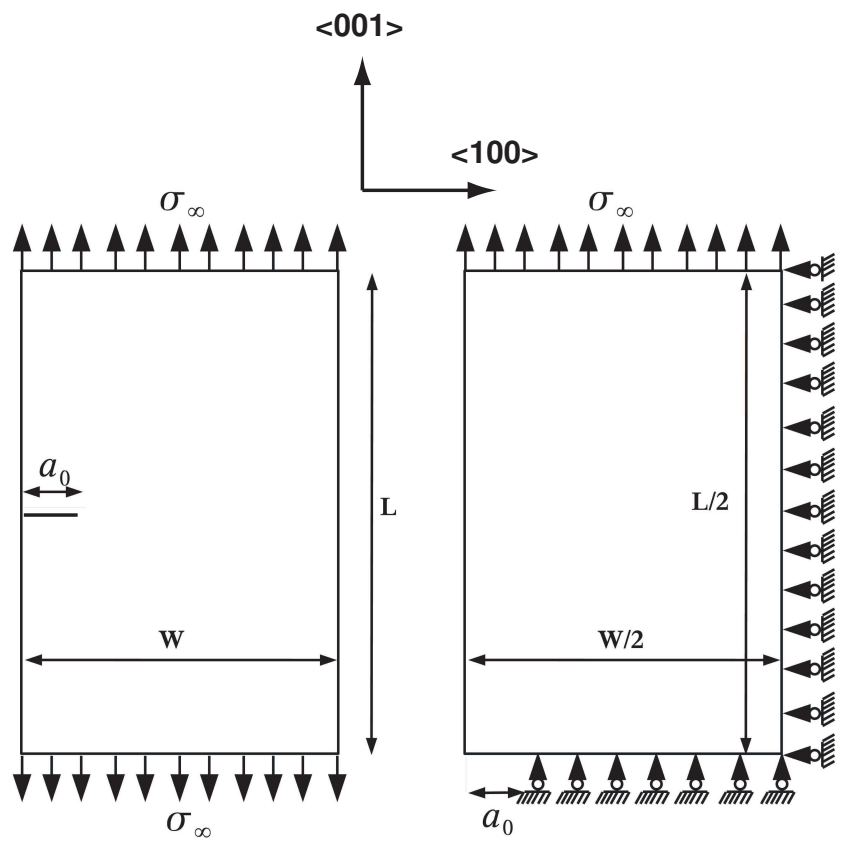

Figure 5. Dimension de l'éprouvette préfissurée, géométrie et conditions aux limites utilisées dans le modèle éléments finis

\subsection{Propriétés du matériau}

Nous présentons ci-après le modèle utilisé pour décrire le comportement élastoviscoplastique d'un monocristal cubique faces centrées développé dans Méric (Meric et al., 1991). Ce modèle, établi dans le cas des petites déformations, est basé sur la théorie de la plasticité cristalline. La déformation totale se décompose en une partie élastique ( $\tilde{\widetilde{C}}$ étant le tenseur anisotrope du module d'élasticité) et une partie plastique :

$$
\tilde{\varepsilon}=\tilde{\varepsilon}^{e}+\tilde{\varepsilon}^{p}, \quad \tilde{\sigma}=\tilde{\tilde{C}}: \tilde{\varepsilon}^{e}
$$

La déformation plastique est la résultante de l'activation des plans de glissement sur les 12 sytèmes octahédriques et les 6 systèmes cubiques. Cette activation des plans de glissement est fonction du chargement appliqué et de l'orientation cristallographique du matériau. On a donc : 


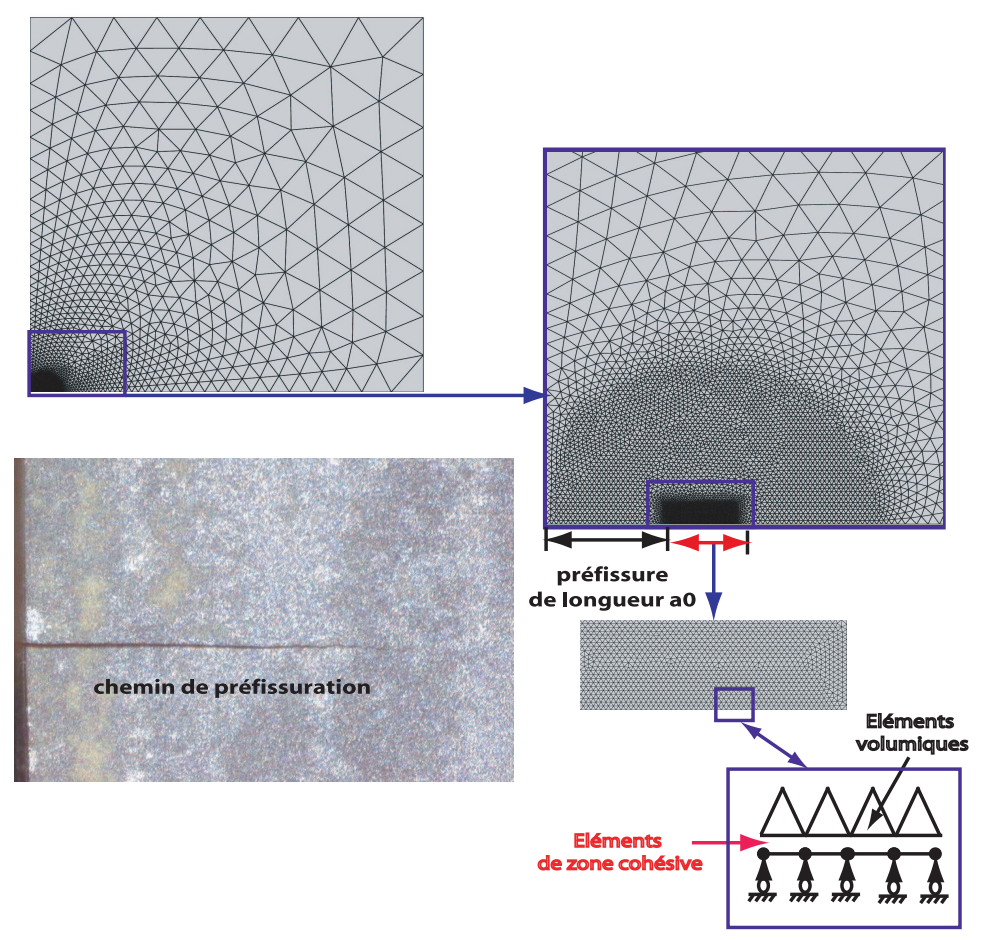

Figure 6. Maillage utilisé
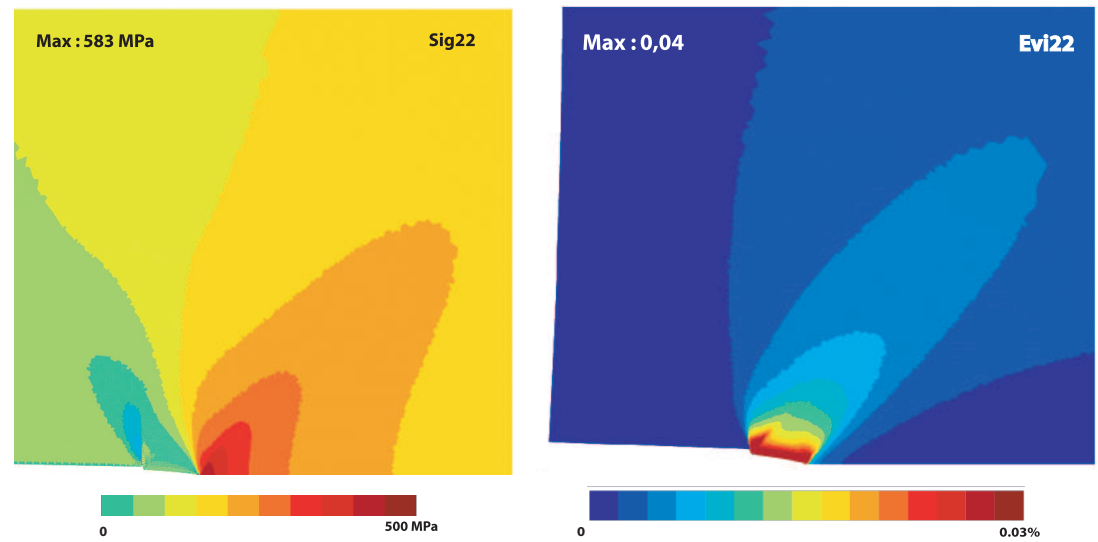

Figure 7. Localisation des champs de contraintes et de déformations plastiques en pointe de fissure sans simulation de la préfissuration 


$$
\dot{\tilde{\varepsilon}}^{p}=\sum_{s=1}^{12} \tilde{m}^{s} \dot{\gamma}^{s}+\sum_{s^{\prime}=1}^{6} \tilde{m}^{s^{\prime}} \dot{\gamma}^{s^{\prime}} \quad \text { avec } \quad \tilde{m}^{s}=\frac{1}{2}\left(\underline{l}^{s} \otimes \underline{n}^{s}+\underline{n}^{s} \otimes \underline{l}^{s}\right)
$$

où $\underline{l}^{s}$ est la direction de glissement et $\underline{n}^{s}$ la normale au plan de glissement, pour le sytème considéré $\mathrm{s}$.

La résultante des vitesses de glissement $\dot{\gamma}^{s}$ est donnée par :

$$
\dot{\gamma}^{s}=\left\langle\frac{\left|\tau^{s}-x^{s}\right|-r^{s}}{k}\right\rangle^{n} \operatorname{sign}\left(\tau^{s}-x^{s}\right)
$$

où $\tau^{s}=\tilde{\sigma}: \tilde{m}^{s}$ est la contrainte de cisaillement résolue sur le plan de glissement s (critère de Schmid). Des variables internes sont introduites pour chaque système de glissement afin de traduire le caractère écrouissable du matériau : les variables d'écrouissage isotrope $r^{s}$ et d'écrouissage cinématique $x^{s}$. Pour l'écrouissage isotrope, la matrice d'intéraction des systèmes de glissement $H^{s r}$ est choisie égale à l'identité.

$$
r^{s}=r_{0}+Q \sum_{r} H^{s r}\left(1-e^{b v^{r}}\right) \quad \text { avec } \quad \dot{v}^{r}=\left|\dot{\gamma}^{r}\right|
$$

La formulation suivante est retenue pour l'écrouissage cinématique non linéaire :

$$
\dot{x}^{s}=c \dot{\gamma}^{s}-\dot{v}^{s} d\left(x^{s}-\bar{x}\right)
$$

La dissymétrie en traction/compression, observée dans les superalliages monocristallins à haute température, est introduite dans l'équation [9] via le terme constant $\bar{x}$. Ce paramètre représente les contraintes internes initiallement présentes dans le matériau. En ne considérant qu'un seul système de glissement, l'intégration de l'équation [9] sous des tractions (+) et compressions (-) monotones donne :

$$
x=\left(\bar{x} \pm \frac{c}{d}\right)\left(1-e^{\mp d \gamma}\right)
$$

Nous résumons, dans le tableau 1 , les différents paramètres du matériau introduits dans la loi de comportement. 


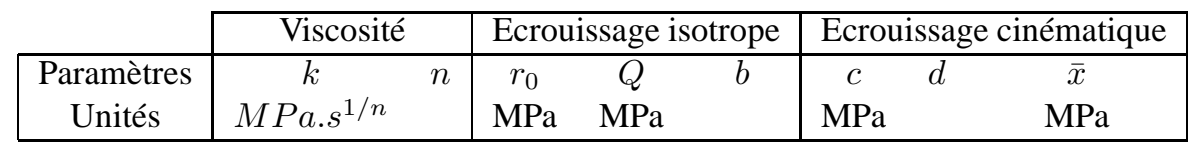

Tableau 1. Récapitulatif des différents paramètres de la loi de comportement cristallographique

\subsection{Validation de l'approche adoptée et simualtion numérique de la propagation de fissure}

Afin de garantir la robustesse des calculs, nous devons nous assurer que la solution converge indépendamment de la taille des éléments d'interface utilisés dans le calcul éléments finis.

Pour la présente étude, nous considérons une analyse à $950^{\circ} \mathrm{C}$ de la propagation de fissure, en mode I, sous chargement cyclique ( $\mathrm{f}=5 \mathrm{~Hz})$, avec le maillage présenté précédemment et en considérant différentes tailles d'éléments (2 à 8 microns).

La figure 8 présente l'évolution de la vitesse de propagation de la fissure en fonction des différentes tailles d'éléments choisies. Ces vitesses de fissuration sont calculées à partir des différentes courbes d'avancée de fissure obtenues pour les différentes tailles d'éléments considérées et ceux pour une longueur de fissure donnée. A partir d'une taille d'éléments de 4 microns, on remarque que la convergence est atteinte et que l'approche zone cohésive est alors indépendante du maillage. Cette dernière taille d'éléments est donc choisie pour les analyses éléments finis présentées ultérieurement.

L'approche adoptée pour simuler la propagation d'une fissure ayant été validée, nous présentons les résultats de la simulation de la propagation d'une fissure dans le cas d'une éprouvette préfissurée (préfissure de 1mm).

Nous pouvons observer, figure 9, les champs de contraintes et de déformations plastiques en pointe de fissure. On remarque que la traînée plastique, mémoire de l'histoire plastique engendrée par l'avancée de fissure sous chargement cyclique est tout à fait régulière. La méthode de relachement de nœuds réalisée pour simuler la préfissure assure ainsi une estimation satisfaisante de la trainée plastique.

La figure 10 donne les évolutions de l'endommagement et de la contrainte le long du chemin de fissuration au maximum de sollicitation des différents cycles. On observe alors que l'évolution de l'endommagement est continue sur le chemin de fissuration. La solution élastique analytique en pointe de fissure a également été tracée. On 


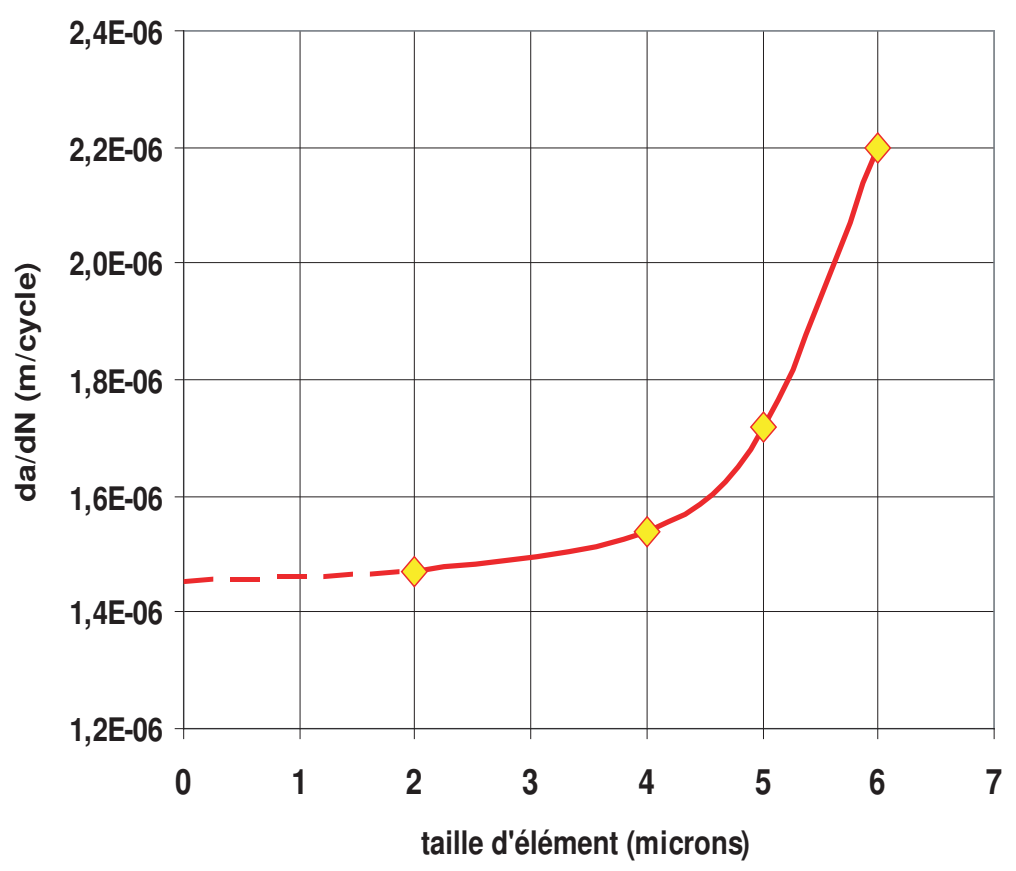

Figure 8. Etude de convergence : influence de la taille des éléments sur la vitesse de fissuration

remarque alors que l'on a une bonne discrétisation, par les éléments de zone cohésive, des contraintes en pointe fissure. La qualité de cette discrétisation est à relier au nombre d'éléments de zone cohésive présents dans la process zone.

\section{Calage du modèle de zone cohésive en régime de fatigue pure}

Dans cette section, nous présentons la méthodologie adoptée pour identifier les paramètres du modèle de zone cohésive sur des essais de fissuration en régime de fatigue pure.

Cette méthodologie se décompose en deux parties :

- identification de la taille de la process zone,

- calibration des paramètres de zone cohésive en régime de Paris.

Nous précisons, dans la suite de cette étude, le rôle joué par les différents paramètres du modèle de zone cohésive. 


\section{Evi 22, $\max : 0,016$}
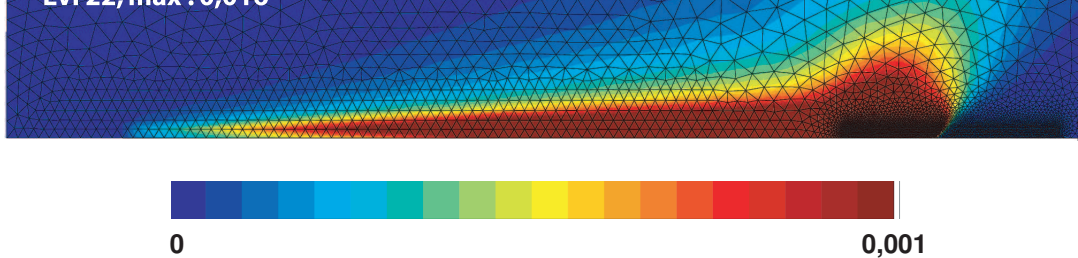

\section{Sig 22, $\max : 2157 \mathrm{MPa}$}

0

$1500 \mathrm{MPa}$

Figure 9. Champs de contrainte, de déformations plastiques en pointe de fissure

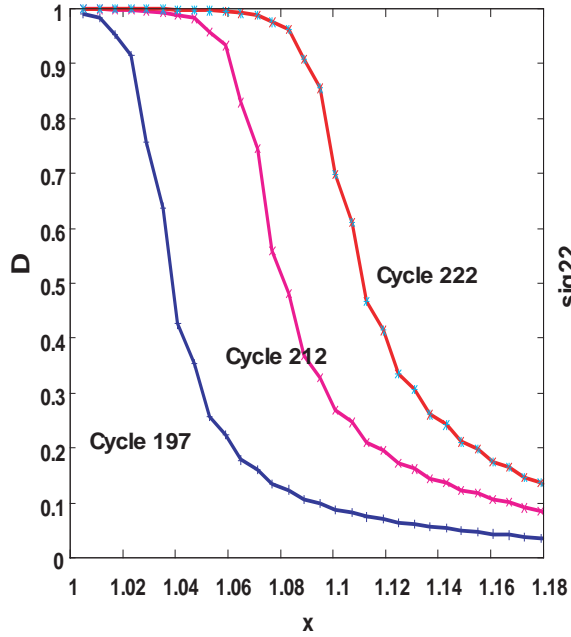

(a)

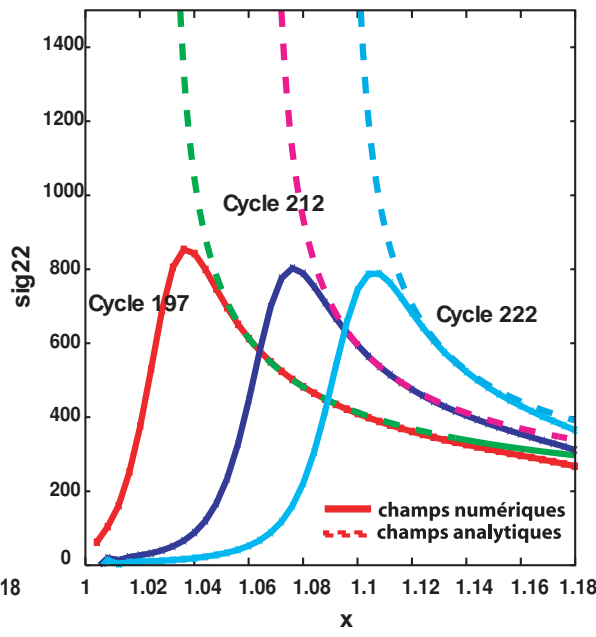

(b)

Figure 10. Evolutions de l'endommagement de fatigue et du champ de contrainte le long du chemin de fissuration à différents cycles 


\subsection{Longueur de la process zone}

L'identification de la taille de la process zone est réalisée en ajustant les paramètres $m, \frac{k_{N}}{\delta_{0}}$ et $T_{0}$. On observe, figure 11 et figure 12 , que le paramètre m permet de contrôler la longueur de la process zone dans la région correspondant à la wake zone. Ainsi, si l'on trace le champ de contrainte le long du chemin de fissuration, on constate que le nombre d'éléments de zone cohésive dans la wake zone augmente lorsque $\mathrm{m}$ augmente.

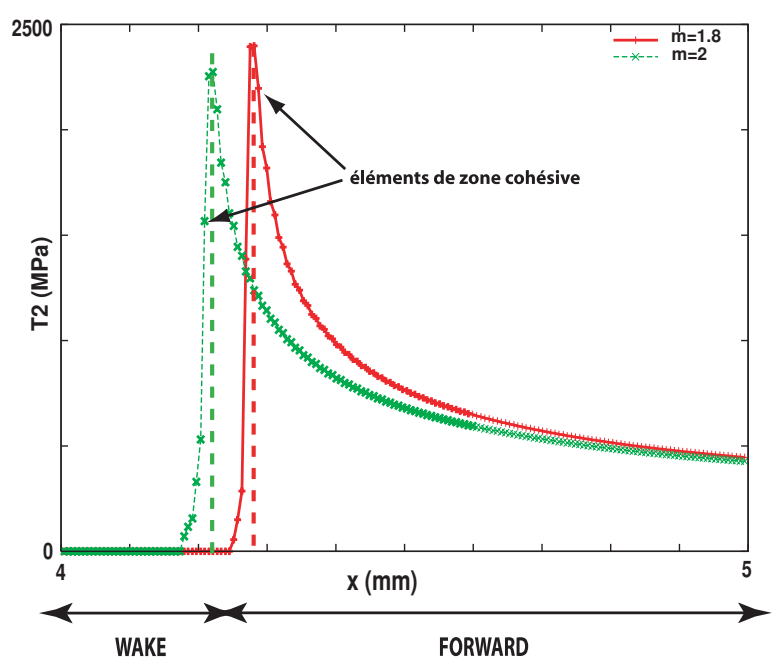

Figure 11. Infuence du paramètre $m$ sur la longueur de process zone le long du chemin de fissuration

Le paramètre $T_{0}$, représentant le seuil de non-propagation, permet, quant à lui, de contrôler la longueur de la forward zone. La figure 13, décrivant l'évolution de l'endommagement le long du chemin de fissuration, montre que plus la valeur de $T_{0}$ est importante, plus la longueur de la forward zone diminue. Une estimation de la longueur de cette forward zone peut être donnée par analogie à la taille de la zone plastique en pointe de fissure. Dans le cas des déformations planes, cette estimation est donnée par :

$$
r_{F Z}=\frac{(1-2 \nu)}{2 \pi}\left(\frac{K_{I}}{T_{0}}\right)^{2}
$$

où $\nu$ est le coefficient de poisson et $K_{I}=\sigma_{\infty} \sqrt{\pi a}$ est le facteur d'intensité des contraintes pour une longueur de fissure donnée a. 


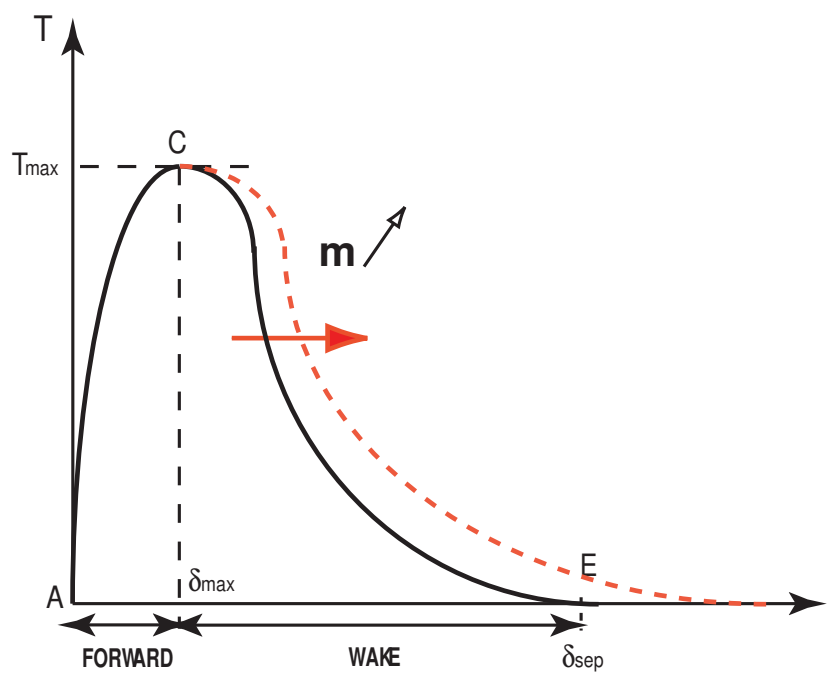

Figure 12. Infuence du paramètre $m$ sur la longueur de process zone

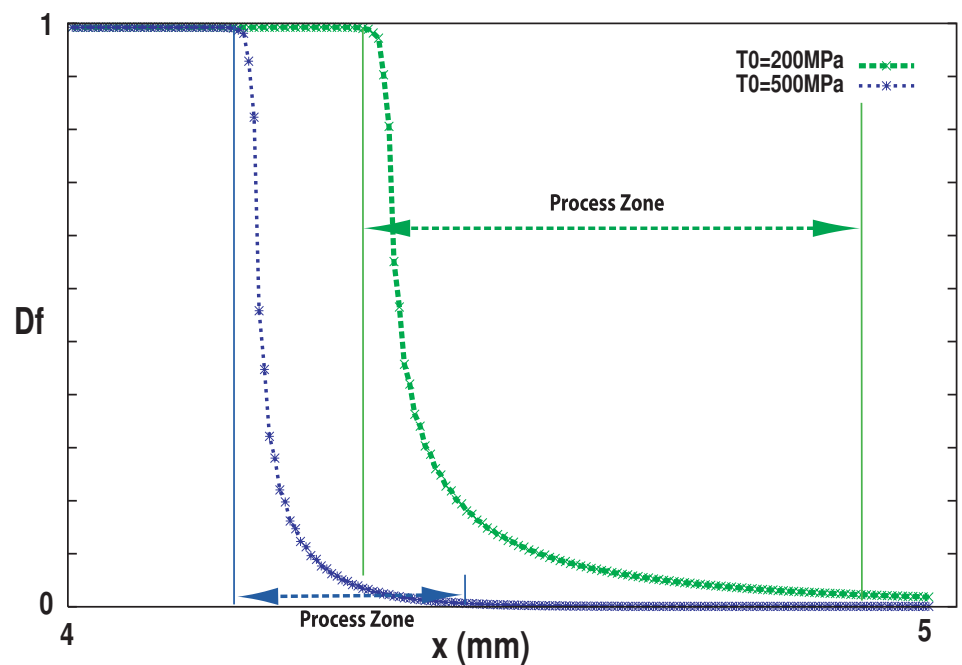

Figure 13. Influence du paramètre de non-propagation, $T_{0}$, sur la process zone

Les paramètres $k_{N}$ et $\delta_{0}$ permettent, non seulement, de déterminer la rigidité initiale de l'élément de zone cohésive mais aussi de contrôler la longueur de la process zone, comme cela est illustré sur cette même figure. Cependant, les paramètres $k_{N}$ et 
$\delta_{0}$ ne permettent pas de modifier indépendamment les tailles de la wake zone et de la forward zone.

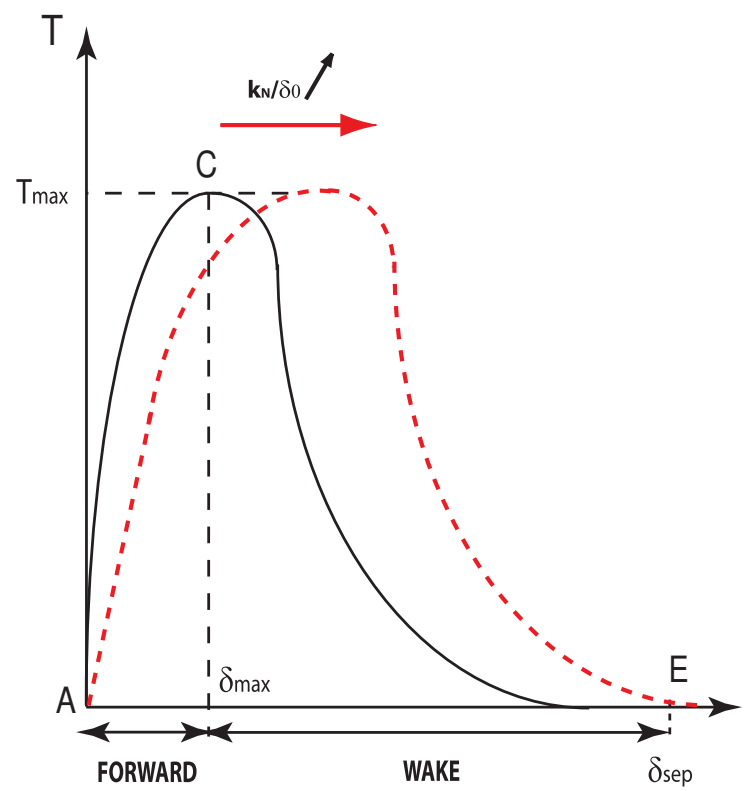

Figure 14. Rôle joué par les paramètres $k$ et $\delta_{0}$

\subsection{Identification des paramètres de zone cohésive en régime de Paris}

Dans le but de comprendre l'influence des paramètres du modèle de zone cohésive sur la vitesse de propagation de fissure en régime de fatigue, nous avons calculé la vitesse d'endommagement $\frac{d D_{f}}{d N}$ en intégrant l'équation [4] au cours d'un cycle :

$$
\begin{aligned}
& \left(1-D_{f}\right)^{-m} \frac{d D_{f}}{d t}=A\left(\frac{k_{N}}{\delta_{0}} \delta-T_{0}\right)^{n} \dot{\delta} \\
& \int_{t(N)}^{t(N+1)}\left(1-D_{f}\right)^{-m} \frac{d D_{f}}{d t} d t=\int_{t(N)}^{t(N+1)} A\left(\frac{k_{N}}{\delta_{0}} \delta-T_{0}\right)^{n} \dot{\delta} d t
\end{aligned}
$$

soit,

$$
\int_{D(N)}^{D(N+1)}\left(1-D_{f}\right)^{-m} d D_{f}=A \cdot \int_{\delta(N)}^{\delta(N+1)}\left(\frac{k_{N}}{\delta_{0}} \delta-T_{0}\right)^{n} d \delta
$$


En supposant que la dérivée $\frac{d D_{f}}{d N}$ évolue peu en fonction du nombre de cycles, comme cela est le cas pour la fatigue à grand nombre de cycles, il est raisonnable d'écrire que :

$$
D_{f}(N+\Delta N) \approx D_{f}(N)+\frac{d D_{f}}{d N} \Delta N
$$

On obtient alors :

$$
\left[1-D_{f}(N+1)\right]^{1-m} \simeq\left(1-D_{f}(N)\right)^{1-m}\left(1+(1-m) \frac{d D_{f}}{d N} \frac{1}{1-D_{f}(N)}\right)
$$

La vitesse d'endommagement au cours des cycles de fatigue est alors donnée par :

$$
\frac{d D_{f}}{d N}=\frac{A}{n+1}\left(1-D_{f}(N)\right)^{m}\left(\left(\frac{k_{N} \delta_{\max }}{\delta_{0}}-T_{0}\right)^{n+1}-\left(\frac{k_{N} \delta_{\min }}{\delta_{0}}-T_{0}\right)^{n+1}\right)
$$

où $\delta_{\max }$ et $\delta_{\min }$ sont respectivement le maximun et le minimum du déplacement à ouverture $\delta$ au cours du cycle.

Des calculs éléments finis ont été réalisés pour différents choix de paramètres du modèle et cela pour deux niveaux de chargements (200MPa et 400MPa) dans le but de calibrer le modèle de zone cohésive avec les données expérimentales. La figure 15 illustre les différentes droites de Paris obtenues par simulations numériques. On remarque que les paramètres $\mathrm{A}$ et $\mathrm{n}$ ont une influence importante dans l'identification de la droite de Paris. Ainsi A permet de translater la droite de Paris et $\mathrm{n}$ de contrôler la pente et ceci en accord avec l'équation [17].

\section{Conclusions}

La simulation numérique de propagation de fissure dans les superalliages monocristallins par des approches couplées reste encore peu étudiée. Nous avons développé et validé une méthode d'avancée de fissure, utilisant les éléments de zone cohésive sous chargement cyclique, à haute température. Le modèle rend compte, avec un caractère prédictif, de l'endommagement progressif des éléments en pointe de fissure, en cohérence avec les champs de contraintes et de déformations (trainée plastique) sous chargement de fatigue, tout en assurant la convergence du calcul à partir d'une taille 


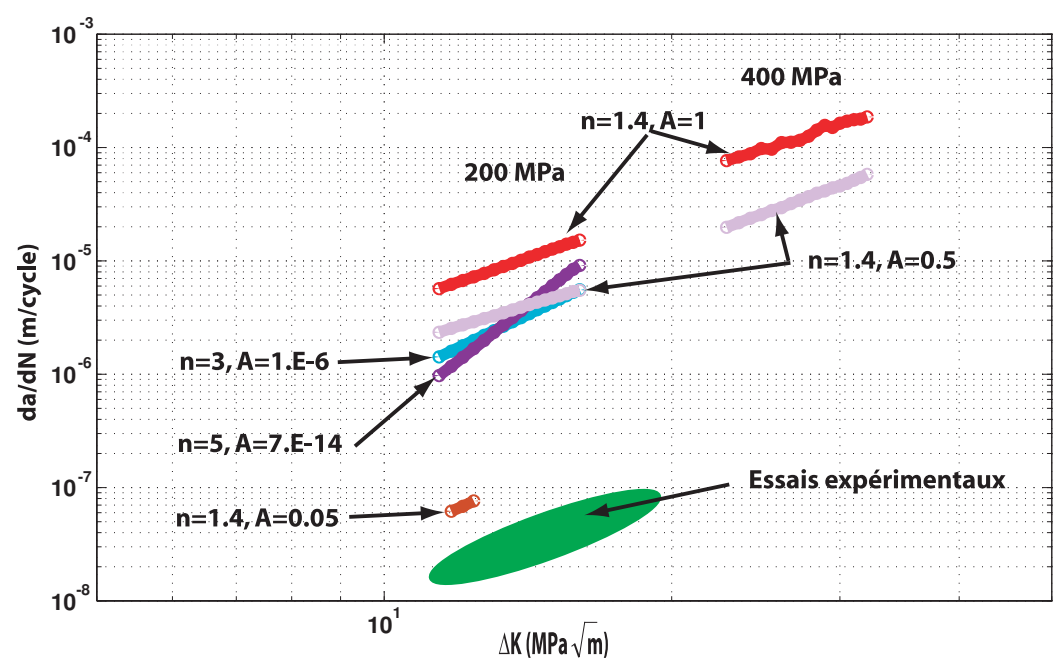

Figure 15. Rôle des différents paramètres dans l'identification de la loi de Paris

d'élément donnée. Il nous faudra par la suite vérifier que les effets de surcharge et de rapport de charge, indispensables à modéliser en fatigue, sont correctement prédits par ce type de modèle. Une méthodologie d'identification des paramètres du modèle de zone cohésive a été établie, le calage de ces paramètres sur la base d'essais obtenus à $950^{\circ} \mathrm{C}$ en régime de fatigue et fatigue-fluage est en cours. Cependant, le modèle d'endommagement doit être encore étendu pour rendre compte des effets d'environnement à haute température (oxydation) ou encore de la redistribution des contraintes, en pointe de fissure, notamment en régime de fluage. Ceci sera traduit, d'une part, par la viscoplasticité du matériau et l'établissement d'une loi d'endommagement de fluage spécifique à l'élément d'interface. D'autre part, les phénomènes d'oxydation seront décrits par l'ajout d'un déplacement $U_{o x}$ dans l'élément de zone cohésive (effets de fermeture de fissure induits par l'oxydation des lèvres) et également par la fragilisation du matériau en pointe de fissure (diminution de la tenacité), ces deux phénomènes étant régis par des cinétiques spécifiques à ce type de superalliage et à ces deux mécanismes.

\section{Remerciements}

Cette étude bénéficie des fonds de la Commission européenne au travers du projet Socrax « Expanding the limits of single crystal superalloys through short crack fracture mechanics analysis ». 


\section{Bibliographie}

Barenblatt G., « The mathematical theory of equilibrium cracks in brittle fracture », Adv. Appl. Mech., vol. 7, p. 55-129, 1962.

Chaboche J., Gallerneau F., « An overview of the damage approach of durability modelling at elevated temperature », Fatigue and Fracture of Engng. Materials and Structures, vol. 24, p. 405-418, 2001.

Chandra N., Shet C., « Analysis of energy balance when using cohesive zone models to simulate fracture processes », Journal of Eng. Mat. Techn., vol. 124, p. 440-450, 2002.

De-Andres A., Perez J., Ortiz M., « Elastoplastic finite element analysis of three-dimensional fatigue crack growth in aluminium shaft subjected to axial loading », Int. J. Of Solids And Struct, vol. 36, p. 2231-2258, 1999.

Dugdale D., « Yielding of steel sheets containing slits », J. Mech. Phys. Solids, vol. 8, p. 100104, 1960.

Meric L., Poubanne P., Cailletaud G., « Single crystal modelling for structural calculations : Part 1-Model presentation », J. Eng. Mat. Techn., vol. 113, p. 162-170, 1991.

Needleman A., « A continuum model for void nucleation by inclusion debonding », Journal of Applied Mechanics, vol. 54, p. 525-531, 1987.

Nguyen Q., Repetto E., M. M. O., Radovitzky, « A cohesive model of fatigue crack growth », Int. J. Of Fract, vol. 110, p. 351-369, 2001.

Paris P., Gomez M., Anderson W., « A rational analytic theory of fatigue », The trend in engineering, vol. 13, p. 9-14, 1961.

Pommier S., Amorçage et propagation de fissures courtes en fond d'entaille. Etude du superalliage N18 en fatigue-fluage, Thèse de doctorat, Ecole Centrale de Paris, 1995.

Prigent P., Modèle de propagation de fissure à haute température avec intéraction fatigue-fluageoxydation, Thèse de doctorat, Ecole Nationale des Ponts et Chaussées, 1993.

Roe K., Siegmund T., « An irreversible cohesive zone model for interface fatigue crack growth simulation », Eng. Fract. Mech., vol. 70, p. 209-232, 2003.

Tate N., A finite element implementation of cohesive zone theory, $\mathrm{PhD}$ thesis, Imperial College London, 2002.

Yang B., Mall S., Ravi-Chandar K., « A cohesive zone model for fatigue crack growth in quasibrittle materials », Int. J. Of Solids And Struct, vol. 38, p. 3927-3944, 2001. 
